\title{
Lesson of the month: Severe leukoencephalopathy by SARS-CoV-2
}

\author{
Authors: Leticia Sanz-Canalejas, ${ }^{A}$ María del Carmen Polidura-Arruga, ${ }^{B}$ Miguel Yus-Fuertes, ${ }^{B}$ \\ Manuela Jorquera-Moya, ${ }^{B}$ Nieves Vidart-Simón, ${ }^{C}$ Juan Arrazola-García ${ }^{D}$ and Natividad Gómez-Ruiz ${ }^{B}$
}

Neurological manifestations associated with SARS-CoV-2 infection as well as its pathogenesis are insufficiently explained. We present two cases of severe COVID-19 who required hospitalisation in the intensive care unit with persistently depressed mental status and severe leukoencephalopathy. We discuss the clinical and radiological findings and also propose the possible pathogenesis involved.

KEYWORDS: COVID-19, coronavirus, leukoencephalopathy, brain, MRI

DOI: $10.7861 /$ clinmed.2020-0940

\section{Introduction}

The pathology of COVID-19, a new infectious disease, and its long-term damage are gradually being described. Scientific knowledge about the neurological involvement of the disease is currently not sufficient. We present two cases of COVID-19 and severe leukoencephalopathy in magnetic resonance imaging (MRI). We discuss the aetiology of these clinical and radiological findings, the neurotropism of SARS-CoV-2 and the pathogenesis of possible neurological infection.

\section{Case report}

Patient 1

A 54-year-old woman with morbid obesity and hypertension was admitted in our hospital with a working diagnosis of communityacquired pneumonia by SARS-CoV-2 that required intensive care unit (ICU) admission. She was managed with mechanical ventilation, intravenous sedation, neuromuscular blockers, prone positioning, vasopressor drugs and renal replacement therapy. She was treated with ritonavir-lopinavir, peginterferon alpha, hydroxychloroquine, ceftriaxone and clarithromycin.

Authors: ${ }^{\text {A }}$ senior radiology resident, Clínico San Carlos University Hospital, Madrid, Spain; ${ }^{\mathrm{B}}$ specialist registrar in neuroradiology, Clínico San Carlos University Hospital, Madrid, Spain; ${ }^{C_{\text {specialist }}}$ registrar in intensive care medicine, Clínico San Carlos University Hospital, Madrid, Spain; ${ }^{D}$ professor and head of department of radiology, Clínico San Carlos University Hospital, Madrid, Spain
Her clinical status improved, although her mental status was persistently depressed despite the withdrawal of sedation. Magnetic resonance imaging (MRI) of the brain was performed and showed bilateral, symmetrical and confluent white matter hyperintense areas on both fast spin echo T2-weighted and fluidattenuation inversion recovery (FLAIR), which were consistent with leukoencephalopathy (Fig 1). Lumbar puncture was not performed. Due to her clinical improvement she was moved to her reference hospital. Unfortunately, it has not been possible to perform a follow-up of any neurological long-term damage.

Patient 2

A 63-year-old man with chronic ischaemic heart disease was admitted to our hospital due to SARS-CoV-2 pneumonia. His

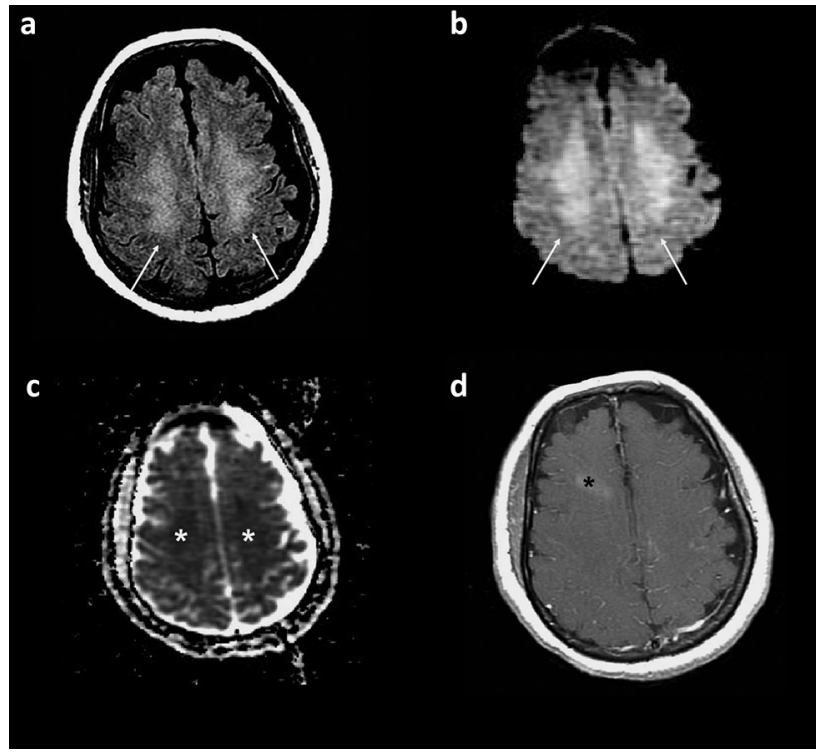

Fig 1. 1.5T magnetic resonance imaging of the brain. Faint bilateral and symmetrical, confluent frontoparietal lesions in centrum semiovale were noted on both fluid-attenuation inversion recovery T2-weighted imaging ( $\mathrm{a}$; arrows) and diffusion-weighted imaging (b; arrows) as hyperintense areas, with diffusion restriction on the apparent diffusion coefficient map (c; asterisks). d) No contrast enhancement fast spin echo T1-weighted imaging; a ghosting artifact is present (asterisk). 


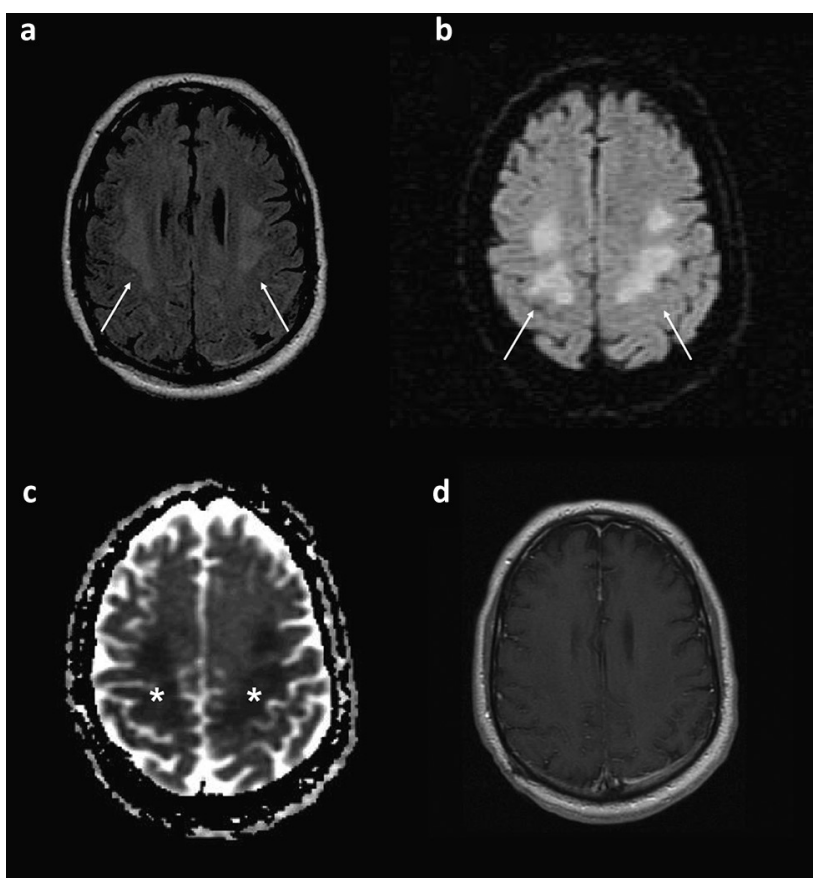

Fig 2. 1.5T magnetic resonance imaging of the brain. Faint bilateral, symmetrical and confluent frontal lesions in centrum semiovale and corona radiata were noted both on fluid-attenuation inversion recovery T2-weighted imaging (a; arrows) and diffusion-weighted imaging (b; arrows) as hyperintense areas, with diffusion restriction on the apparent diffusion coefficient map (c; asterisks). d) No contrast enhancement fast spin echo T1-weighted imaging.

condition worsened and required ICU admission. He was managed with the general measures of the critical patient with multiple organ failure and SARS-CoV-2 infection. His progression was favourable, so the sedation was withdrawn. Because his mental status was persistently depressed, MRI of the brain was performed and showed identical findings to those described in our previous case (Fig 2). Lumbar puncture was not performed. He was discharged and unfortunately it has not been possible to perform a follow-up of any neurological long-term damage.

\section{Discussion}

From the neurological point of view, SARS-CoV-2 has been associated with ischaemic and haemorrhagic strokes, acute necrotising haemorrhagic encephalitis, stupor, coma and delirium in addition to headache, anosmia and ageusia. ${ }^{1}$ Up to $36 \%$ of patients with COVID-19 have neurological manifestations, being more frequent in patients with greater clinical severity. In critical patients with COVID-19, neurological symptoms usually involves several aetiological factors (such as sedation drugs, hypoxic and metabolic encephalopathy, encephalopathy induced by sepsis, and the potential neurological damage induced by the virus itself or by para-infectious mechanisms). ${ }^{1}$ Therefore, it is unlikely that a single isolated aetiological factor triggered the severe leukoencephalopathy of our patients.

It is well known that some betacoronaviruses are able to infect the brain of animals and humans. ${ }^{2,3}$ Neurons, and also glial and endothelial cells of the cerebral microcirculation are susceptible to infection by SARS-CoV-2 because they have angiotensin converting enzyme 2 receptors on their cell surface. ${ }^{1,4}$ Like any other pathogen with neurotropism, SARS-CoV-2 could infect the central nervous system using three well-known mechanisms. First, the blood-brain barrier altered by hypoxia and an inflammatory storm could favour the passage of the virus. ${ }^{5}$ The second mechanism is retrograde axonal infection, shown for SARS-CoV in a murine animal model through olfactory bulbs. ${ }^{6}$ Finally, the virus could access the brain inside infected immune cells ('Trojan horse' mechanism). ${ }^{7}$ SARS-CoV-2 has been detected in brain tissue by RT-PCR and electron microscopy and also in cerebrospinal fluid samples. ${ }^{7,8}$

Cerebrospinal fluid analysis and histopathology has shown lack of a strong inflammatory response associated to brain infection by SARS-CoV-2. ${ }^{6,8}$ COVID-19 lymphopenia is a marker of severity and an important prognostic factor. ${ }^{9}$ In autopsies of patients who died from SARS, there was a significant atrophy of the mucosa-associated lymphoid tissue (MALT), lymph nodes and spleen. ${ }^{10}$ Gu et al proposed that patients with the most severe forms of infections developed a rapidly progressive acquired cellular immunodeficiency and, in the late stages of infection, it would favour the spread of the virus in different organs with a low lymphocyte tissue infiltration.

\section{Summary}

We present two cases of severe leukoencephalopathy associated with SARS-CoV-2 in critically ill patients. Many questions remain to be resolved relating to COVID-19 and its long-term outcome, including potentially disabling neurological involvement.

Therefore, scientific research, complementary tests (like MRI) and autopsies remain essential to improve morbidity and mortality associated with COVID-19.

\section{Key points}

> COVID-19 often involves lungs, however, up to $36 \%$ of these patients have neurological manifestations, being more frequent in severe cases.

$>$ We describe and provide images of two cases of COVID-19 with severe leukoencephalopathy.

$>$ We discuss the neurotropism and the pathogenesis of possible neurological infection by SARS-CoV-2.

> These cases showed the systemic and probable brain infection by SARS-CoV-2 and emphasises the importance of neurological evaluation and clinical follow-up of these patients.

\section{References}

1 Zhou Z, Kang H, Li S, Zhao X. Understanding the neurotropic characteristics of SARS-CoV-2: from neurological manifestations of COVID-19 to potential neurotropic mechanisms. J Neurol 2020;267:2179-84

2 Li YC, Bai WZ, Hashikawa T. The neuroinvasive potential of SARS-CoV2 may play a role in the respiratory failure of COVID-19 patients. J Med Virol 2020;92:552-5.

3 Yashavantha Rao HC, Jayabaskaran C. The emergence of a novel coronavirus (SARS-CoV-2) disease and their neuroinvasive propensity may affect in COVID-19 patients. J Med Virol 2020;92:786-90.

4 Mankad K, Perry MD, Mirsky DM, Rossi A. COVID-19: A primer for Neuroradiologists. Neuroradiology 2020;62:647-8. 
5 Zhengqian L, Taotao L, Ning Y et al. Neurological manifestations of patients with COVID-19: potential routes of SARS-CoV-2 neuroinvasion from the periphery to the brain. Front Med 2020;14:533-41.

6 Netland J, Meyerholz DK, Moore S, Cassell M, Perlman S. Severe acute respiratory syndrome coronavirus infection causes neuronal death in the absence of encephalitis in mice transgenic for human ACE2. J Virol 2008:82:7264-75.

7 Paniz-Mondolfi A, Bryce C, Grimes Z et al. Central nervous system involvement by severe acute respiratory syndrome coronavirus-2 (SARS-CoV-2). J Med Virol 2020;92:699-702.

8 Moriguchi T, Harii N, Goto ] et al. A first case of meningitis/ encephalitis associated with SARS-Coronavirus-2. Int I Infect Dis 2020;94:55-8.
9 Terpos E, Ntanasis-Stathopoulos I, Elalamy I et al. Hematological findings and complications of COVID-19. Am J Hematol 2020;95: 834-47.

10 Gu J, Gong E, Zhang B et al. Multiple organ infection and the pathogenesis of SARS. J Exp Med 2005;202:415-24.

Address for correspondence: Dr Leticia Sanz-Canalejas, Radiology Department, Clínico San Carlos University Hospital, Calle del Profesor Martín Lagos, 28040 Madrid, Spain.

Email: leticia.sanzcanalejas48@gmail.com 